\title{
La resolución de problemas y el pensamiento numérico en los procesos de enseñanza- aprendizaje significativos de la división
}

\section{Problem Solving Numerical and Thought Processes in Teaching-learning Division Significant}

\author{
Sandra Peña Alonso*
}

Fecha de recepción: 19 de octubre de 2009

Fecha de aprobación: 23 de octubre de 2009

\section{Resumen}

Este proyecto de investigación responde a la necesidad pedagógica, didáctica y disciplinar de brindar a los estudiantes del curso $6^{\circ} \mathrm{B}$ del Colegio Rafael María Carrasquilla (Bogotá, localidad 18) alternativas para generar un aprendizaje significativo en el proceso de la división y de su concepto fundamental de reparto. Para tal fin se elaboró una cartilla en la que se presentan situaciones problemáticas, creadas a partir de diferentes experiencias en la escuela, las cuales constituyen el insumo necesario para que los educandos conciban la construcción de su conocimiento como una fuente de incertidumbres, reflexiones, preguntas, conflictos y espacios de socialización.

Palabras clave: problema, resolución de problemas, estructuras multiplicativas, división, repartos.

Egresada Programa de Educación Básica con énfasis en Matemáticas, de la Facultad de Educación, Universidad Santo Tomás de la Vicerrectoría de Universidad Abierta y a Distancia (VUAD), Bogotá. Colombia. Correo electrónico: sandy070283@yahoo.es 


\section{Abstract}

This investigation project answers to the pedagogical, didactic and discipline needs to offer to the students of the $6^{\circ} \mathrm{B}$ grade (Rafael Maria Carrasquilla school, Bogotá, locality 18) alternatives for provide meaningful learning into division process and his fundamental concept. As a result was elaborated a primer where are presented problematic situations created from different experiences at school, which constitute the necessary input so that the pupils understand the construction of their knowledge as a source of uncertainties, reflections, questions, conflicts and spaces of socialization.

Key words: problem, problems resolution, division, multiplication structures, distributions.

\section{Problema}

\section{Problemática}

Al asumir la responsabilidad de formar en Matemáticas el grupo de niños y niñas del curso $6^{\circ} \mathrm{B}$, durante el transcurso del año escolar 2006, fue posible apreciar que los estudiantes presentaban falencias de comprensión en varios de los procesos propuestos en el currículo institucional, específicamente el de la división, cuando resolvían problemas aritméticos. Al comienzo fue tan solo una apreciación subjetiva, pero con el pasar del tiempo y con el desarrollo de la temática se hizo evidente que los educandos, fuera de tener dificultades en el manejo y dominio del algoritmo de la división, no comprendían los diferentes términos que intervienen en esta operación (dividendo, divisor, residuo, cociente) y mucho menos las posibles relaciones que se establecen entre éstos.

Por otra parte, las metodologías de enseñanza utilizadas buscaban que los estudiantes aprendieran en un ambiente contextualizado y para lograrlo en este proceso se involucraron las situaciones problema, con las cuales resultaba más difícil orientar el desarrollo de competencias interpretativas, argumentativas y propositivas; lo anterior no fue posible, pues para los niños representaba mayor complejidad el hecho de pensar la división desde un problema que, además de utilizar el algoritmo, requería el manejo de otros elementos de carácter comunicativo, como son: la comprensión lectora, la extracción de datos, el planteamiento y la aplicación de estrategias para buscar soluciones a situaciones que no siempre contaban con una estructura que se pudiera resolver en forma directa y para lo cual ellos manifestaban no sentirse preparados.

Al finalizar la fase de diagnóstico se encontró que los niños a nivel del pensamiento numérico presentaban dificultades para:

- Dar un significado a los términos de las operaciones realizadas como estrategia de 
solución a un problema y a la manera como éstos se relacionan.

- Al multiplicar no ubicaban correctamente los números según su valor posicional y terminaban multiplicando inicialmente decenas con unidades, unidades con decenas, o decenas con decenas.

- No trabajaban los algoritmos en una estructura diferente al esquema tradicional o vertical y una multiplicación en forma horizontal representaba un verdadero problema.

En cuanto a la resolución de problemas que involucraban acciones de reparto se evidenció que:

- Los niños tenían un bajo nivel de comprensión en la lectura de situaciones problema, se hacía necesario que el docente leyera para todo el grupo y luego hiciera una explicación de lo leído.

- No había un manejo adecuado al leer los signos de puntuación, interrogación y admiración dentro de la situación problema.

- No había un orden lógico o coherente para organizar la información existente en un problema al igual que para extraer sus datos.

- Los niños no utilizaban el dibujo como una ayuda para ilustrar sus ideas y evidenciar sus niveles de comprensión acerca de una situación problema.

- Los estudiantes proponían estrategias en las que se evidenciaba claramente que no manejan un orden lógico y coherente en cuanto a las acciones por seguir y así buscar diferentes soluciones a un mismo problema.
- La atención que prestaban a la pregunta dentro del problema era mínima, no tenía mayor trascendencia.

- Las estrategias de solución que proponían los estudiantes para resolver un problema no permitían visualizar mayor reflexión y planeación, pues estas propuestas involucraban únicamente la aplicación de una operación.

- La estrategia de solución de los niños terminaba en el momento que resolvían la operación matemática. El resultado obtenido después de esta acción se convertía en la solución inmediata del problema.

\section{Conclusiones generales del diagnóstico}

- Los niños asocian intrínsecamente el concepto de dividir con el de repartir.

- Cuando los niños llevan un recorrido amplio en el proceso de la división trabajan en forma más elaborada los conceptos de todo parte, parte de una parte, parte de un todo.

- El principio de los repartos es el concepto de mitad y mitad de la mitad.

- Los repartos se constituyen como un todo a través de la suma.

- El material tangible como los palillos es tomado como una representación real de una idea abstracta que ya poseen los niños.

- Los niños manifiestan abiertamente que dividir es repartir en forma equitativa. Es fraccionar.

- En cuanto a la resolución de problemas no todas las situaciones problema tienen la misma estructura, por eso la búsqueda 
de estrategias de solución siempre varía dependiendo el contexto inicial de la situación y el contexto en la que el niño desee resolverla.

- Cada niño tiene un ritmo de aprendizaje individual y único, es importante identificar las características generales de los estilos de aprendizaje del grupo en general respetando las diferencias para llevarlos a progresar simultáneamente.

- Las situaciones que implican problema activan esquemas mentales de pensamiento en los niños.

- Aunque el problema se le presente como problema a los niños ellos lo reducen a un ejercicio mecánico en el que operan con suma, resta, multiplicación y división aunque no tengan conciencia de por qué lo hacen. La aplicación de operaciones no implica necesariamente la solución a un problema.

\section{Planteamiento del problema}

¿Cómo hacer de la resolución de problemas una herramienta para dotar de significado y sentido los procesos de enseñanza-aprendizaje de la división?

\section{Objetivos}

\section{Objetivo general}

Diseñar una propuesta pedagógica, didáctica y disciplinar que trabaje desde su estructura el problema de la significación del aprendizaje de la división, enmarcado dentro del contexto de la resolución de problemas.

\section{Objetivos específicos}

- Propiciar espacios de auto-reflexión en los cuales los niños tengan la oportunidad de reconocer sus habilidades, capacidades y limitantes en la resolución de problemas.

- Fomentar el diálogo como una herramienta para generar múltiples estrategias de solución a problemas que involucran la división.

- Promover la práctica de valores sociales como el respeto, la responsabilidad, la tolerancia y la solidaridad al trabajar en grupo.

- Desarrollar en los niños procesos de pensamiento numérico comprensivo y contextualizado en situaciones problémicas.

- Dotar de sentido y significación las operaciones matemáticas enmarcadas en el algoritmo de la división y la resolución de problemas que las involucren directa o indirectamente.

- Abordar el problema del aprendizaje de la división en los niños, a partir del planteamiento de situaciones problémicas.

- Brindar las herramientas necesarias para que los niños se inicien en la reconstrucción de conceptos como el de reparto para llegar a la resignificación de las operaciones matemáticas, especialmente de la división, implementando la solución de problemas.

\section{Justificación}

Atendiendo a las necesidades e intereses de los estudiantes del grado $6^{\circ} \mathrm{B}$ de poder resignificar su aprendizaje de las operaciones matemáticas, específicamente de sus estructuras multiplicativas (división) en el desarrollo 
del pensamiento numérico, se hace necesario crear una propuesta de intervención en la cual ellos, como dinamizadores de su proceso y de su crecimiento intelectual, sean capaces de lograr relaciones entre los conceptos y los procedimientos que ya conocen; de tal manera que encuentren diversas maneras de operar con miras a la resolución de situaciones problema.

Según se propone en los lineamientos curriculares, es necesario dotar de sentido y significado las operaciones aritméticas, como la división durante el proceso de enseñanza-aprendizaje, ya que estas acciones pedagógicas y didácticas dan la pauta para que el estudiante reconozca el significado de cada operación en situaciones concretas, en las cuales tienen origen, por ejemplo, en la división la acción de repartir de manera equitativa diferentes cantidades continuas y discontinuas, para que reconozca los modelos más usuales de cada operación como los funcionales, métricos, lineales, entre otros, para que haga una verdadera comprensión de las diferentes propiedades que se cumplen en cada operación. Finalmente, para que los estudiantes estén en capacidad de hacer alusión a su dominio conceptual y a los procesos cognitivos inherentes a la actividad matemática de la que participan.

Por otra parte, el desarrollo del pensamiento numérico depende de la variedad de reflexiones que el docente pueda generar en torno a las relaciones que se establecen entre las operaciones en cada una de sus estructuras y la manera como éstas inciden en la búsqueda de cálculos o estimaciones numéricas aunque se sabe que:

\begin{abstract}
La finalidad de los cálculos es la resolución de problemas. Por lo tanto, aunque el cálculo sea importante para las matemáticas y la vida diaria, la era tecnológica en que se vive obliga a replantear la forma en que se utiliza el cálculo hoy día (Lineamientos Curriculares de Matemáticas, 1998).
\end{abstract}

Teniendo en cuenta que los educandos en su proceso de formación atribuyen diversos significados al concepto de número y a su aplicación en el desarrollo de operaciones, es la resolución de problemas lo que sirve como medio para que este tipo de relaciones se establezcan y, en consecuencia, permitan el alcance de los fines institucionales que se proponen en el currículo porque:

- El estudiante está en capacidad de plantear problemas con las operaciones adecuadas $y$, a la vez, utilizar diversas estrategias para resolverlos.

- Los educandos cuentan con las herramientas necesarias para enfrentarse a los problemas menos rutinarios y más abiertos a soluciones múltiples.

- Los educandos desarrollan habilidades comunicativas que facilitan el trabajo en equipo en beneficio de su aprendizaje, habilidades de razonamiento y argumentación por medio de las cuales estarán en la facultad de dar razón al porqué de su proceder frente a un problema.

La resolución de problemas como contexto que permite resignifcar y dotar de sentido operaciones aritméticas básicas como la división, ayudan para que el docente: 
- Genere ambientes en los cuales los estudiantes exploren, se inquieten, sientan curiosidad y se sientan motivados a realizar una tarea.

- Presente situaciones variadas y contextualizadas para brindar a los estudiantes la posibilidad de observar, jerarquizar, clasificar, describir, transformar información, hacer inferencias y preguntas acerca de una situación problema.

- Promueva el uso de modelos en los cuales se apoyen los niños, como presaberes, para que sean los ingenieros y constructores de otros conocimientos más significativos por la utilidad que representan a la hora de facilitar una tarea en la resolución de un problema.

- Participe e interactúe con los niños en la vivencia de valores sociales como la tolerancia, la cooperación, la colaboración, la solidaridad y la responsabilidad.

- Prepare a sus educandos para que sean autónomos en la toma de decisiones responsables, dejando el precedente de que toda decisión trae consigo sus propias consecuencias.

Con la vinculación de estrategias y metodologías en la propuesta de intervención se espera que los estudiantes resuelvan situaciones problema, para que:

- Ellos sean competentes independientemente de sus formas individuales de abordar un problema, no quedándose sólo en la estrategia, sino también en el avance hacia el planteamiento de soluciones eficaces.
- Los análisis que hagan los estudiantes de una misma situación de reparto permitan establecer relaciones numéricas en función de la operación de la división y el debido procesamiento de la información disponible.

- Los estudiantes muestren grandes avances en el dominio de su estructura multiplicativa al finalizar la educación básica, al reconocer la función lineal y sus propiedades como "una herramienta más potente en el tratamiento de situaciones de tipo multiplicativo".

- Que los educandos establezcan relaciones con otros conceptos matemáticos comenzando por el de división, razón, proporción, proporcionalidad y función lineal.

\section{Elementos teóricos}

\section{Pensamiento numérico}

Los niños lo adquieren gradualmente y evoluciona en la medida en que ellos tienen la oportunidad de pensar los números y de usarlos en contextos significativos. Incluye el desarrollo de tres capacidades fundamentales.

- Comprensión de los números y la numeración: es un proceso sistemático que se inicia con la construcción de significados y con la posterior caracterización del sistema de numeración. Es fundamental que los niños comprendan el significado de los números, sus diferentes interpretaciones y sus representaciones, así como las formas como se utilizan para contar, para expresar una cantidad, para medir como secuencia verbal, como cardinal o como tecla para pulsar. 
- Comprensión del concepto de las operaciones: este proceso incluye las destrezas relacionadas con reconocimiento del significado de las operaciones en situaciones concretas e hipotéticas, el reconocimiento de los modelos más usuales y prácticos de las operaciones, lo mismo que la comprensión de las propiedades y el efecto al aplicar cada operación.

- Cálculo con números y aplicación de números y operaciones: tradicionalmente, este proceso ha recibido un mayor énfasis en la formación básica. En este sentido, se orienta hacia la comprensión de las operaciones, se debe prescindir del conocimiento matemático reducido al manejo de algoritmos formales y enfocarlo hacia el desarrollo del cálculo mental.

\section{La división}

La división es una operación aritmética en la cual el resultado es un par ordenado de números que indica el número de veces que es posible repartir una cantidad denominada dividendo en otra llamada divisor. Al establecerse esta relación de términos se transforman las cantidades involucradas para obtener dos cantidades más y en las cuales una de éstas representa el número de elementos que se distribuyen equitativamente en cada reparto y se conoce con el nombre de cociente. En algunas situaciones que se presentan bajo otras condiciones, quedan elementos sin repartir, porque no son suficientes para completar otro reparto y esta última cantidad se denomina residuo. Otra forma de comprender la división es cuando se presenta un número llamado $A$ y se pide sea repartido entre un número $B$ que significa las veces que puede ser restado $B$ de $A$, encontrándose así un tercer elemento denominado $\mathrm{C}$ que determina el número de veces que se restó $B$ de $A$.

\section{Los algoritmos}

Son maneras estandarizadas de operar, son métodos que se llevan a cabo paso a paso, en los que se presentan los datos de entrada, en un orden establecido para llegar a obtener finalmente un dato de salida o resultado. E algoritmo tiene un carácter global y permite la transformación de información.

Un algoritmo tiene las siguientes características:

- Es finito: para buscar una solución operativa a un problema se ejecutan determinado número de pasos. Se conserva un orden para obtener de la misma manera un resultado.

- Debe estar definido: las acciones que se ejecutan para obtener un resultado poseen un orden preestablecido. En caso de que se presenten en más de una oportunidad los mismos datos de entrada al aplicar el algoritmo se deben obtener los mismos datos de salida.

\section{El problema}

Es una tarea para la cual los individuos que se enfrentan a ésta deben encontrar una solución al realizar un procedimiento que no es de fácil acceso y para lo cual son ellos quienes deben buscar el camino que más los acerque o de respuesta. 
El problema en sí se genera cuando no se tiene la respuesta efectiva a una situación dada. La persona asume una conducta que interactúa con sus sistemas de procesamiento de la información de acuerdo con la estructura del ambiente de la tarea, los espacios del problema y la información incluida en los espacios del problema.

Los problemas según su estructura pueden ser:

- Bien definidos: son aquellas situaciones en las que en el enunciado se muestra de forma clara la relación entre los datos de entrada y los de salida, poniendo en evidencia con anticipación las operaciones que se van a ejecutar para obtener la solución.

- Mal definidos: son aquellas situaciones en las que los pasos son menos claros, al igual que su punto de partida, puede tener varias soluciones, es difícil para la persona que lo resuelve saber o tener la certeza de haber alcanzado la solución. No muestra los pasos ni las operaciones para concebir planes en busca de la solución.

Para el psicólogo Wertheimer (1945), cuando un problema está bien definido el individuo pone en práctica un pensamiento reproductivo que consiste en la aplicación de estrategias y de métodos ya conocidos, mientras que ante un problema mal definido se hace necesario que se ponga en práctica un pensamiento productivo que incluya la búsqueda de formas de solución nuevas, al organizar y reorganizar los elementos del problema en función de la solución.
Algunos factores que intervienen en el proceso de resolución de un problema (Schoenfeld, 1992) son los siguientes:

El conocimiento base

Hace referencia a los conocimientos previos o herramientas conceptuales o procedimentales con las que cuenta el niño a la hora de resolver un problema. El conocimiento previo o base, inicialmente, puede ser intuitivo e informal; hace parte de este tipo de conocimiento los hechos, las definiciones, los procedimientos algorítmicos, los procedimientos rutinarios y no rutinarios y el uso del lenguaje.

El conocimiento base o de recursos es específicamente el vocabulario y las estrategias de solución a situaciones rutinarias y no rutinarias.

\section{Las estrategias o heurísticas}

Según Polya, las heurísticas son métodos generales que se aplican en la resolución de un problema independiente del contenido de cada situación, como: 
- Comprensión del problema: se debe identificar el grado de dificultad que tiene la situación para la persona, quien la resuelve esperando de esta manera una actitud de superación frente a ésta.

\begin{tabular}{|c|c|}
\hline $\begin{array}{c}\text { COMPRENDER } \\
\text { UN PROBLEMA } \\
\text { IMPLICA }\end{array}$ & $\begin{array}{l}\text { Entender las palabras que son desconocidas en el enunciado. } \\
\text { Traducir el leguaje en el que se presenta el problema a uno más } \\
\text { accequible por el individuo. } \\
\text { Identificar el significado de los símbolos. } \\
\text { Asumir la situación como conflictiva. } \\
\text { Disposición para la búsqueda de soluciones. }\end{array}$ \\
\hline $\begin{array}{c}\text { COMPRENDER } \\
\text { UN PROBLEMA } \\
\text { IMPLICA }\end{array}$ & $\begin{array}{l}\text { Saber cuál es la incógnita o necesidad. } \\
\text { Identificar las condiciones del problema (bien definido-mal } \\
\text { definido). } \\
\text { ¿Cuáles son los datos? } \\
\text { ¿Es posible relacionar estos datos? }\end{array}$ \\
\hline
\end{tabular}

- Diseñar un plan: puede comprender estrategias, reglas, algoritmos, operaciones. Una estrategia depende de lo que el individuo considere es la necesidad más relevante dentro de la situación, pues lo que para una persona es un problema para otra no lo es.

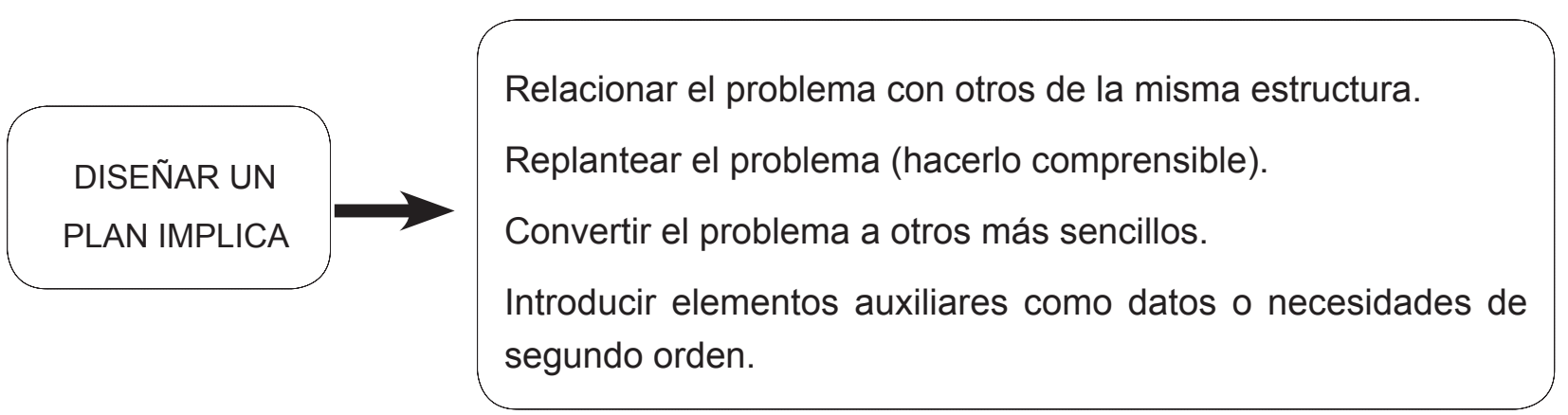

- Examinar los resultados o soluciones: se debe evaluar o valorar el alcance de las metas propuestas en el plan de acción en un primer momento, y luego es necesario ser consciente de la riqueza de las estrategias y reglas aplicadas para lograr la solución. 
- La evaluación incluye hacer una reflexión sobre la manera como el individuo se dispuso frente al problema al inicio, durante su desarrollo y en la solución.

\begin{tabular}{|l}
$\begin{array}{c}\text { EL EXAMEN DE } \\
\text { LA SOLUCIÓN } \\
\text { ENCONTRADA } \\
\text { IMPLICA }\end{array}$ \\
Mroblema. \\
Preguntarse si es posible usar el mismo método para solucionar \\
otros problemas.
\end{tabular}

La resolución de problemas implica fundamentalmente:

- Que la persona comprenda el problema y haga una traducción de éste a símbolos matemáticos.

- La programación de estrategias para hallar una solución final requiere de técnicas más amplias.

Otros factores que intervienen en el proceso de resolución de problemas es:

- Los aspectos metacognitivos: hacen alusión a la manera como el estudiante selecciona y hace uso de los recursos matemáticas y de las estrategias de las que dispone en la búsqueda de soluciones. Al finalizar y al haber encontrado la solución a su problema, el educando evalúa sus fortalezas, debilidades y establece claramente los aspectos por mejorar frente a determinadas problemáticas. Hacer metacognición es tener la capacidad para monitorear y controlar la manera como se resuelve un problema.

- Sistemas de creencias: cada individuo, a parte de lograr un nivel elevado de conceptualización y de simbolización de algunas acciones matemáticas, después de resolver un problema ha logrado vincular afectivamente sus intereses y necesidades en función de estos saberes. La actitud, la afectividad, la necesidad de hacer algo en beneficio de un mejor aprendizaje hacen que los estudiantes se vean motivados a realizar tareas cada vez más complejas.

El simple hecho de no conocer la respuesta a determinada pregunta ya genera incertidumbre, inconformismo e intranquilidad. Es así como algunas concepciones tienen relación directa con la resolución de problemas y es la de frustración y miedo al fracaso. Varias personas desisten de resolver un problema antes de interiorizarlo, porque consideran que no están lo suficientemente preparadas, muestran una predisposición tan grande que el tiempo que asignan a la búsqueda de una solución es mínimo, en ocasiones hasta se llega a carecer de iniciativa para proponer estrategias o formas de solución.

Para algunos individuos designar tiempo para realizar una planeación resulta ser una actividad dispendiosa; es en este momento cuando deciden empezar a dar pasos rápidos y cortos que se convierten en estrategias mínimas que se van consolidando a partir del ensayo y el error. 


\section{Metodología}

La investigación se realizó en tres fases: diagnóstico, plan de acción, aplicación de la propuesta y análisis de los resultados con un grupo de 26 niños. Se hizo uso de la observación directa, la entrevista, la encuesta y de dos formatos de exploración. Con el primero se buscaba identificar el dominio y la manera como los estudiantes entendían la acción de repartir y la forma como la relacionaban con el concepto de división; en el segundo se presentaron cuatro situaciones problema que implicaban el uso de la división para evidenciar cómo procedían de los estudiantes frente a este tipo de estructuras.

Durante el proceso, el estudiante es visto como sujeto que aprende y participa de la enseñanza de las operaciones aritméticas básicas específicamente. Se toma como referencia su forma de resolver problemas que implican división; es el estudiante y sus comprensiones en la resolución de problemas el objeto sobre el cual se realiza todo el estudio. Se parte de una observación directa y participante de los educandos en su contexto escolar, sin desconocer otros factores que puedan llegar a incidir en su manera de aprender, de ahí que en la recolección de la información se haga un énfasis especial en la manera como se han venido familiarizando con el uso de operaciones como la división y su forma especifica de proceder frente a una situación problema.

\section{La propuesta}

Se creó una cartilla en formato impreso y virtual en el cual, a partir de tres procesos matemáticos: comunicación, razonamiento y resolución de problemas, se proponían una serie de situaciones problémicas con diversas estructuras que incluían desde un enunciado, una tabla o un diagrama de barras hasta situaciones en las que se hacía necesario su construcción a partir de un grupo de datos, y otras en la que era necesario extraer, incluir y buscar otros.

La cartilla tiene una estructura que inicia con el planteamiento del propósito de la sesión o encuentro, el reconocimiento de presaberes, continúa con la presentación de los conceptos y los procedimientos clave y luego con problemas que involucran los tres procesos antes mencionados. Al abordar cada proceso también se buscaba que los estudiantes hagan uso de representaciones gráficas y simbólicas. Las situaciones problema fueron construidas a partir de elementos, personajes, anécdotas, actividades y vivencias que se experimentan a diario en la institución educativa.

\section{Principales hallazgos}

En el contexto de la resolución de problemas:

- La pregunta es uno de los elementos del problema al que más valor le dan los estudiantes, pues manifiestan que sin ésta no tiene sentido resolver el problema, porque deja de representar una situación de conflicto. 
- Las dificultades, en cuanto a la comprensión lectora de un problema, fueron superadas a medida que los niños adquirieron las bases conceptuales de algunos significados, conocieron los elementos de un problema, aprendieron a extraer e incluir datos, a plantear y extraer necesidades, habilidades que representaron una ganancia a la hora de leer una situación, pues ya tenían unos conceptos previos que sirvieron como soporte para lograr mejores niveles de comprensión.

- Los estudiantes deben leer más de una vez el problema para identificar la necesidad cuando no se presenta de manera explicita.

- Los niños comprenden mejor un problema cuando es el docente quien se los lee.

- Los signos de puntuación, a pesar de que no son bien leídos ni acentuados, son parte del enunciado que no se puede relegar a un segundo puesto, manifiestan los estudiantes. Porque la lectura errada de un signo cambia total o parcialmente la comprensión de una situación problema y, por tanto, la planeación de estrategias y soluciones; en consecuencia, no serían las más adecuadas.

- Los estudiantes planifican sus estrategias, comenzando por la formulación de una operación aritmética.

- La división como operación cobró sentido en la medida en que los estudiantes recurrían a su uso para solucionar situaciones que directamente la involucran.
En el contexto de la división:

- Hay un buen dominio y manejo del algoritmo de la división en la mayoría de los estudiantes.

- Los estudiantes reconocen la relación existente de los términos de la división sólo en algunas situaciones problema.

- Hay dificultades en cuanto al manejo de la división por dos cifras o cuando el dividendo es menor que el divisor.

- Algunos niños evitan dividir y realizan cálculos mentales que después verifican a partir de la multiplicación.

\section{Análisis de los resultados}

\section{Categoría 1: resolución de problemas}

\section{El concepto de problema}

Inicialmente, los niños creían que un problema era sólo aquella situación o acontecimiento que se refería exclusivamente a la aplicación de operaciones matemáticas. Este concepto lo fueron ampliando y modificando en la medida en que se fue trabajando en el reconocimiento de situaciones problema de diferente carácter, unas matemáticas, otras cotidianas, otras de las que surgen en la escuela, etc.

\section{Los elementos del problema}

Los estudiantes desconocían qué elementos conformaban la estructura de un problema. A partir de la sesión número uno, se empezó a tra- 
bajar para superar esta dificultad. Después de la segunda sesión los estudiantes ya estaban en capacidad de nombrar e identificar dentro de un problema sus elementos. Posteriormente, se propuso a los estudiantes la inclusión y la extracción de estos elementos del problema, como es el caso de los datos y las necesidades. Se observa que al finalizar el proceso los estudiantes aún tienen dificultad para identificar el contexto que rodea la situación problema. Esto obedece a que todos los problemas fueron elaborados en diferentes contextos.

Son los datos la fuente de información más valiosa para los educandos, porque consideran que si no hay datos específicamente numéricos, deja de ser un problema aritmético y, por tanto, su solución dejaría de tener cabida desde el pensamiento matemático.

\section{La estructura de los problemas}

Para los niños fue una gran sorpresa el primer día cuando empezaron a leer y encontraron en las situaciones redactadas muchas de sus experiencias y con el nombre de algunos de ellos. A parte de la sorpresa, los niños se sintieron motivados a buscar el problema en el cual iban a intervenir; fue motivo de risa y alegría, encontrar su vida de escolares reflejada en enunciados, los cuales, aparte de brindarles una información, les estaba generando la oportunidad de aprender.

En cuanto a las situaciones que traían consigo una tabla o diagrama de barras, se evidenció que los estudiantes son muy ágiles extrayendo datos. Para ellos representó un reto no tener toda la información a su alcance, pues manifes- taban que así pensaban más y además ponían a prueba sus habilidades con respecto al tema.

Los problemas que se presentaron en desorden total fueron de los que más disonancia cognitiva generaron en los educandos, puesto que ellos asimilaron que el problema era como un rompecabezas que debían construir y del cual sólo conocían algunas de sus partes, más no su contenido. El hecho de construir el problema para luego leerlo y empezar el proceso de solución, ya representaba una situación problemática, puesto que varios de ellos optaron por organizarse con sus compañeros para que cada uno hiciera una propuesta y entre ellos seleccionan la mejor, de acuerdo con su criterio.

Por otra parte, los problemas que carecían de algún elemento como la necesidad o los datos representaron menos dificultad para los niños, ya que para ellos fue sencillo iniciar leyendo la situación e ir incluyendo lo que hiciera falta de acuerdo con el contexto. Algunos lograron establecer una forma lógica del problema de acuerdo con la información conocida y desconocida.

La pregunta en el problema

Los estudiantes consideraban que sin la pregunta se quedarían sin herramientas para saber qué solucionar. Es más, no habría nada que solucionar, porque sin preguntas no hay problema. La utilidad que le atribuyeron a la pregunta sirvió para concluir que es la pregunta la que indica y deja la sensación de inquietud, de intranquilidad, es la clave para solucionar el problema. 
LA PREGUNTA

EN EL

PROBLEMA

Conduce a la solución.

Expresa una necesidad que se debe suplir.

Complementa la información existente.

Sirve como base para planear la estrategia.

Facilita la comprensión del problema.

La creación de problemas

Se observa que cuando los elementos del problema están dados antes de crear un problema, los niños tienden a organizarlos en el orden que se presentan. Las necesidades las expresan a través de preguntas al finalizar la situación.

Es de vital importancia la presentación de datos numéricos e imágenes a la hora de crear un problema, ya que los niños saben aprovechar muy bien estas ayudas. Realizan una transformación de ésta; de manera creativa, llegan a incluir información que no está presente en estos elementos. Se apoyan en situaciones que han vivido o en anécdotas que surgen como recuerdos gratos.

\section{La división como operación}

Entre los problemas, se esperaba que los estudiantes plantearan una división como parte de la estrategia de solución al encontrar un dato o para relacionar la información encontrada y hacer uso de la multiplicación, la suma, la resta y el cálculo numérico por aproximación.

La utilidad de la división como operación se evidenció durante las últimas sesiones; los estudiantes optaron por tomar el algoritmo y aplicarlo como una alternativa para obtener datos que contribuyeran de manera directa a la solución.
La forma de operar es de manera vertical, todos los algoritmos los aplicaban de una manera mecánica, es decir, inicialmente ellos proponían una operación, pero desconocían las razones del porqué lo habían hecho de esa forma y no de otra; en algunos casos y en otros sabían que era una parte de la estrategia, pero no lograban justificar la existencia coherente de una posible relación entre los datos seleccionados en la entrada.

Los estudiantes avanzaron en cuanto al significado que le atribuyen a cada uno de los operadores del algoritmo. Cuando establecen la operación tienen plena conciencia de cuáles son los datos de entrada, muestran confusión en algunas situaciones, porque los datos de salida no satisfacen sus necesidades de solución. Los datos de salida se tornan confusos, ya que en la mayoría de las socializaciones los educandos no mostraron mayor nivel de comprensión de la manera como se pueden relacionar los datos; ellos saben que lo deben hacer en función de la necesidad, pero no logran avanzar en este aspecto.

\section{Categoría 2: procesos matemáticos}

Proceso de comunicación

Se encontró que los estudiantes tienen dificultad para expresar en forma escrita varias de sus 
ideas. Algunas veces omiten el uso de signos de puntuación y de algunas palabras, expresan una pregunta sin signos de interrogación. Al iniciar la aplicación de la propuesta, era común escuchar expresiones como: "profe... ¿qué debo hacer?", después ellos fueron comprendiendo que cada sesión tenía una finalidad y que todas las acciones que se realizaran durante el encuentro estaban orientadas hacia la adquisición de este fin.

Proceso de resolución de problemas

Fue mejorando a medida que se fue evolucionando en el desarrollo de las sesiones; inicialmente, los estudiantes llegaban hasta el reconocimiento de los elementos del problema, después se avanzó hacia la propuesta de diferentes estrategias y finalmente se alcanzó la aplicación de los diferentes planes, y para la verificación se observó que en la medida en que los niños iban aplicando su estrategia iban reflexionando sobre lo que estaba mal en su propuesta.

\section{Categoría 3: formas de representación de la información}

\section{Representación gráfica y simbólica}

Los niños realizan dibujos, gráficas, algunos hacen esquemas para mostrar sus ideas y pensamientos. No todos utilizan el dibujo a color, la mayoría de los niños lo hacen a un solo color de lápiz o esfero; para otros niños es más importante simbolizar y explicar de manera verbal su proceder. Las formas de representación son variadas y tienen un sello de originalidad y de pertenencia por el trabajo realizado.

\section{Conclusiones}

Al finalizar este proyecto de investigación resulta un tanto nostálgico recordar que lo que comenzó como una sencilla reflexión se convirtió con el paso del tiempo y con trabajo, esfuerzo, dedicación y responsabilidad en una realidad, de la cual se puede decir que es la primera de tantas satisfacciones, que se puede tener en el ejercicio docente cuando existe un verdadero compromiso y una libre voluntad de mejorar y aportar al crecimiento y desarrollo de los futuros ciudadanos y profesionales del país.

Esta propuesta, enfocada hacia el desarrollo del pensamiento numérico y los sistemas numéricos, aplicados en el aprendizaje significativo de la división fue evolucionando a medida que se logró generar en los estudiantes valores como la solidaridad, el respeto, la tolerancia, la responsabilidad y la disponibilidad para trabajar en equipo.

El desarrollo del pensamiento numérico se fue dando gradualmente y se fue avanzando en la medida en que los estudiantes tuvieron la oportunidad de construir conceptos como el de división, desde la ejecución de acciones básicas como la de reparto, de pensar y usar contextos reales, para resolver situaciones problema y de generar múltiples y variadas estrategias de solución a éstas. Resultó interesante ver que los niños escogían, desarrollaban y buscaban estrategias para hacer más fácil su trabajo de comprensión en la resolución de problemas.

La resolución de problemas de manera satisfactoria depende de la familiaridad de la 
situación con la vida de los educandos, pues es este factor el que aumenta sustancialmente la capacidad de razonamiento, pues para lograrlo se debe llevar a la práctica partiendo de la libre aplicación de conocimientos previos. Es la experiencia la primera forma de familiarizarse con la resolución de problemas en contextos paralelos al de la educación matemática.

En cuanto al crecimiento intelectual, social y afectivo de los estudiantes, se evidencia en su capacidad para reconocer que tienen grandes capacidades de aprender $\mathrm{y}$, a la vez, admitir que el aprendizaje no se logra si no hay una motivación intrínseca que perdure y vaya más allá del aula, es decir, que trascienda el contexto escolar.

\section{Referencias}

Amore, B. (s.f.). Didáctica de la matemática. Bogotá: Editorial Magisterio.

Autores Varios (1998). Lineamientos curriculares de Matemáticas. (Áreas obligatorias y fundamentales). Bogotá: Editorial Magisterio.

Bermejo, V. (s.f.). Instrucción y construcción de las primeras nociones aritméticas. Ediciones Paidós.

Briones, G. (1998). La investigación social y educativa. Bogotá: Convenio Andrés Bello.
Callejo, M. (s.f.). Un club matemático para la diversidad. Narcea.

Castro, E., Rico, L. \& Castro, E. (1995). Estructuras elementales y su modelización. Bogotá: Grupo Editorial Iberoamericana.

Cerda, H. (2000). La evaluación como experiencia total. Bogotá: Editorial Magisterio.

Dallura, L. (s.f.). La matemática y su didáctica en el primero y segundo ciclos de la E. G. B. Un enfoque Constructivista. Aique.

García O., G. (s.f.). Currículo y evaluación en matemáticas. Bogotá: Editorial Magisterio.

Kopitowski, A. (s.f.). Enseñanza de la Matemática. (Entre el discurso y la práctica). Aique.

Puentes, Y. (2001). Organizaciones escolares inteligentes. Aula abierta. Bogotá: Editorial Magisterio.

Resnick, L. \& Ford, W. (s.f.). La enseñanza de las matemáticas y sus fundamentos psicológicos. Ministerio de Educación y Ciencia.

Sarmiento, M. (1999). Cómo aprender a enseñar y cómo enseñar a aprender: (Psicología educativa y del aprendizaje). Bogotá:

\section{Universidad Santo Tomás.}

